\section{Depressão pós-parto: considerações terminológicas}

\author{
Postpartum depression: terminological \\ considerations
}

\author{
Lucas Spanemberg \\ Especialista em Psiquiatria. Tesoureiro, Centro de Estudos em \\ Psiquiatria Integrada (CENESPI), e psiquiatra, Ambulatório de \\ Auxílio à Cessação do Tabagismo, Hospital São Lucas, Pontifícia \\ Universidade Católica do Rio Grande do Sul (PUCRS), Porto \\ Alegre, RS. Professor convidado, Instituto Fernando Pessoa (IFP), \\ Porto Alegre, RS. \\ Correspondência:1spanemberg@yahoo.com.br
}

Prezados Editores,

Sobre o trabalho intitulado "Aspectos epidemiológicos da depressão pós-parto em amostra brasileira"1, gostaria de tecer algumas críticas em relação à terminologia usada, que pode confundir e gerar falsos dados sobre quadros depressivos.

Em primeiro lugar, é sabido que a literatura especializada difunde uma série de instrumentos diagnósticos (qualitativos) e de gravidade de sintomas (quantitativos) sobre avaliação da doença depressiva ${ }^{2}$. Aos autores dos estudos cabe, então, escolher entre um grande leque de opções, de acordo com critérios que levem em conta o que está sendo avaliado. No caso do presente estudo, utilizou-se uma escala de screening para depressão (Edinburgh Postnatal Depression Scale $\mathrm{EPDS}^{3}$ ), amplamente utilizada na literatura internacional. Contudo, a partir dos resultados do trabalho supracitado, houve confusão terminológica entre "sintomatologia depressiva" (sugestiva do transtorno, mas não definidora) e diagnóstico de depressão pós-parto. Além disso, todas as tabelas apresentadas usaram o diagnóstico "depressão pósparto" ou "mulheres deprimidas versus nãodeprimidas", ao invés de caracterizar o que realmente se estudou, isto é, a sintomatologia depressiva no pósparto. Considerou-se, pois, essa escala como definidora do diagnóstico de depressão pós-parto, o que não é o objetivo da mesma. Os próprios autores da escala sugerem que os escores sugestivos devem passar por uma avaliação clínica cuidadosa para confirmar o diagnóstico ${ }^{3}$.

Em nosso meio, é muito comum que estudos de prevalência usem escalas quantitativas ou de screening para fazer diagnóstico de depressão $0^{4-7}$. Alguns desses estudos acabam descrevendo corretamente o uso dos instrumentos (EPDS, Beck, Hamilton, etc.), mas pecam na descrição dos resultados, considerando os pontos de corte como definidores do diagnóstico. Além disso, pontos de corte diferentes dificultam a comparação dos dados.
Bernardi $^{8}$ alerta nesse mesmo volume sobre os paradoxos gerados pelos avanços no estudo da saúde mental: por um lado, a possibilidade de novos recursos terapêuticos e, por outro, uma possível flexibilização de algumas entidades nosológicas (e tendência à medicalização) por motivos não-científicos. Pode-se ainda considerar que o uso inadequado de escalas pode proporcionar uma superestimação diagnóstica ou ainda uma confusão diagnóstica, uma vez que é comum a sobreposição de sintomas em diferentes categorias diagnósticas.

Deve-se acrescentar que o estudo sobre sintomatologia depressiva no pós-parto com a EPDS é de extrema importância e de elevada pertinência, visto que possibilita uma triagem rápida e simples, que pode ser aplicada por qualquer profissional. A validade de uma abordagem desse tipo justifica-se principalmente em ambientes de atenção primária, onde a maior parte das puérperas é atendida. Porém, pacientes com escores de sintomas sugestivos de depressão devem ser avaliados com abordagens mais completas. Por fim, a literatura científica deve primar pelo uso adequado dos termos técnicos e instrumentos ou corre o risco de difundir informações equivocadas, que contribuem para o já saturado oceano de estatísticas controversas dos nossos dias.

\section{Referências}

1. Ruschi GE, Sun SY, Mattar R, Chambô Filho A, Zandonade E, Lima VJ. Aspectos epidemiológicos da depressão pós-parto em amostra brasileira. Rev Psiq RS. 2007;29(3):274-80.

2. Calil HM, Pires MLN. Aspectos gerais das escalas de avaliação da depressão. Rev Psiq Clin. 1998;25(5):240-4.

3. Cox JL, Holden MJ, Sagovsky R. Detection of postnatal depression: development of the 10-item Edinburgh Postnatal Depression Scale. Br J Psychiatry. 1987; 150:782-6.

4. Furegato ARF, Silva EC, Campos MC, Cassiano RPT. Depressão e auto-estima entre acadêmicos de enfermagem. Rev Psiquiatr Clin. 2006;33(5);239-44.

5. Moraes IG, Pinheiro RT, Silva RA, Horta BL, Sousa PL, Faria AD. Prevalence of postpartum depression and associated factors. Rev Saude Publica. 2006;40(1):65-70.

6. Cruz EBS, Simões GL, Faisal-Cury A. Rastreamento da depressão pós-parto em mulheres atendidas pelo Programa de Saúde da Família. Rev Bras Ginecol Obstet. 2005;27(4):181-8.

7. Da-Silva VA, Moraes-Santos AR, Carvalho MS, Martins ML, Teixeira MA. Prenatal and postnatal depression among low income Brazilian women. Braz J Med Biol Res. 1998;31(6):799-804.

8. Bernardi R. Transtornos de humor bipolar: uma visão integradora. Rev Psiq RS. 2007;29(3):259-61.

\section{Resposta dos autores}

Prezados editores,

Contrastando com outras áreas da medicina, recursos diagnósticos subsidiários são pouco utilizados 
em psiquiatria, especialidade eminentemente clínica ${ }^{1}$. Mesmo o DSM-IV, ao listar os sintomas, não tem o propósito de substituir a abrangência do diagnóstico clínico $^{2}$. A utilização de instrumentos padronizados de avaliação dos sintomas psiquiátricos nas pesquisas clínicas tem se justificado pela obtenção de medidas acuradas e reprodutíveis ${ }^{1}$.

$\mathrm{O}$ artigo intitulado "Aspectos epidemiológicos da depressão pós-parto em amostra brasileira"3 deixa bem claro que a escolha da Escala de Depressão Pós-parto de Edimburgo (EPDS) deveu-se ao fato de ser um instrumento desenvolvido especificamente para aplicação em mulheres no período de 12 meses após o parto. Mesmo havendo grandes variações nos pontos de corte adotados nas validações realizadas em diversos países que se diferenciam cultural e socioeconomicamente do Brasil ${ }^{4}$, o estudo de validação nacional adota pontuação $11 / 12$ por ter apresentado melhores índices de predição ${ }^{5}$.

Apontamos como limitação do estudo o fato da EPDS ser um instrumento de triagem dos sintomas depressivos, não havendo intenção de polemizar e até mesmo desvalorizar o trabalho da equipe de saúde mental. Consideramos as escalas de avaliação instrumentos indispensáveis na Atenção Básica de Saúde de um país carente de profissionais especializados.

Embora tenhamos inserido tabelas e gráficos que se referem à confirmação diagnóstica de depressão na impressão gráfica do artigo, o texto manteve sua coerência ao informar que o instrumento adotado é de triagem diagnóstica, não dispensando a avaliação clínica do especialista.

\section{Referências}

1. Jorge MR, Custódio O. Utilidade das escalas de avaliação para clínicos e pesquisadores. Rev Psiquiatr Clin. 1999;26(2):102-5.

2. Gomes de Matos E, Gomes de Matos TM, Gomes de Matos GM. A importância e as limitações do uso do DSM-IV na prática clínica. Rev Psiquiatr RS. 2005;27(3):312-8.

3. Ruschi GEC, Sun SY, Mattar R, Chambô Filho A, Zandonade E, Lima VJ. Aspectos epidemiológicos da depressão pós-parto. Rev Psiq RS. 2007;29(3):274-80.

4. Halbreich U, Karkun S. Cross-cultural and social diversity of prevalence of postpartum depression and depressive symptoms. J Affect Disord. 2006;91(2-3):97-111.

5. Santos MFS. Depressão pós-parto: validação da Escala de Edimburgo em puérperas brasilienses [tese]. Brasília: Instituto de Psicologia; 1995.

\section{Gustavo Enrico Cabral Ruschi}

Especialista em Ginecologista e Obstetrícia. Mestre em Ciências da Saúde, Área de Concentração: Obstetrícia, Universidade Federal de São Paulo - Escola Paulista de Medicina (UNIFESPEPM), São Paulo, SP. Ginecologista e obstetra, Instituto Estadual de Saúde Pública do Espírito Santo (IESP) e Prefeitura Municipal de Vitória, Vitória, ES. Professor assistente, Programa de Interação Serviço-Ensino-Comunidade (PISEC), Centro Universitário Vila Velha (UVV), Vila Velha, ES

Correspondência: gruschi@terra.com.br 